AB0194

\section{BTLA EXPRESSION IS REDUCED ON SLE B CELL} SUBSETS

Annika Wiedemann ${ }^{1}$, Ana-Luisa Stefanski ${ }^{1,2}$, Andreia Lino ${ }^{1}$, Thomas Dörner ${ }^{1}$. ${ }^{1}$ Charité Universitätsmedizin Berlin, Dept. of Medicine, Rheumatology and Clinical Immunology, Berlin, Germany, ${ }^{2}$ University Hospital of Bern, Department of Rheumatology, Immunology and Allergology, Bern, Switzerland

Background: B- and T-lymphocyte attenuator (BTLA/CD272) is a coreceptor constitutively expressed on B cells $[1,2]$. Upon $B$ cell activation via the $\mathrm{B}$ cell receptor (BCR), BTLA co-localizes with Src homology region 2 domain-containing phosphatase-1 (SHP-1) and thus negatively regulates $B C R$ signaling [2]. A recent study found that $C D 4+T$ cells from SLE patients fail to upregulate BTLA upon activation [3], however, data on BTLA expression and function for B cells in autoimmunity is missing.

Objectives: To assess BTLA expression on conventional naïve $\left(\mathrm{CD} 20^{+} \mathrm{CD} 27^{-}\right)$, conventional memory $\left(\mathrm{CD} 20^{+} \mathrm{CD} 27^{+}\right) \quad \mathrm{B}$ cells and on $\mathrm{CD} 27^{++} \mathrm{CD} 38^{++}$expressing plasma cells (PC) in peripheral blood mononuclear cells (PBMCs) of patients with systemic lupus erythematosus (SLE) and healthy donors (HD).

Methods: PBMCs were isolated from EDTA blood taken from 7 female SLE patients (age 38, mean disease activity 5 (SLEDAl)) and 6 female HD (mean age 28) by Ficoll density gradient centrifugation according to the manufacturer's protocol. Cells have been stained and expression of BTLA was assessed by flow cytometry.

Results: Analysis of BTLA surface expression on B cell subsets in SLE patients and HD revealed decreased expression of BTLA on naïve SLE $B$ cells $\left({ }^{*} p=0.0173\right.$, Mann-Whitney $U$ Test (MWU), BTLA median fluorescence intensity (MFI) 9006 \pm 1450 ) compared to naïve HD B cells (11957 $\pm 941)$. A similar tendency was found for memory SLE B cells $(p=0.0823$ MWU) compared to HD memory but not SLE PC and HD PC. Remarkably, an inverse correlation was found for BTLA expression on naïve SLE B cells and SLE PC with Siglec-1 expression on monocytes $\left({ }^{*} \mathrm{p}=0.0333\right.$ Spearman's rank correlation (SRC) naïve $\mathrm{B}$ cells, ${ }^{*} \mathrm{p}=0.0167$ $\mathrm{PC})$, a marker for interferon signature, and the same trend was seen for SLE memory B cells $(p=0.0583$ SRC). Inverse correlation of BTLA expression was also found with disease activity (SLEDAI) with these B cell populations but did not reach significance $(p=0.0583$ naïve $B$ cells, $p=0.1361$ memory $B$ cells, $p=0.0833 P C$ ).

Conclusion: Herein, we document that B cell subsets of SLE patients express lower levels of the negative regulator BTLA than HD. Additionally, an inverse correlation between BTLA expression on B cell subsets and Siglec-1 on monocytes were found suggesting its involvement in disease and consideration BTLA as therapeutic target in SLE. We hypothesize that reduced BTLA expression is a feature of post-activated B cells. Further studies need to delineate functional properties of BTLA expression and activation in autoimmune B cells.

\section{REFERENCES:}

[1] Watanabe N, Gavrieli M, Sedy JR, Yang J, Fallarino F, Loftin SK, Hurchla MA, Zimmerman N, Sim J, Zang X, et al: BTLA is a lymphocyte inhibitory receptor with similarities to CTLA-4 and PD-1. Nat Immunol 2003, 4(7):670-679.

[2] Vendel AC, Calemine-Fenaux J, Izrael-Tomasevic A, Chauhan V, Arnott $D$, Eaton DL: B and T lymphocyte attenuator regulates B cell receptor signaling by targeting Syk and BLNK. J Immunol 2009, 182(3):15091517.

[3] Sawaf M, Fauny JD, Felten R, Sagez F, Gottenberg JE, Dumortier H, Monneaux F: Defective BTLA functionality is rescued by restoring lipid metabolism in lupus CD4+ T cells. JCl Insight 2018, 3(13).

Disclosure of Interests: Annika Wiedemann: None declared, Ana-Luisa Stefanski: None declared, Andreia Lino: None declared, Thomas Dörner Grant/research support from: Eli Lilly, Janssen, Roche, UCB Pharma, Consultant for: Eli Lilly, Janssen, Roche, UCB Pharma, Speakers bureau: Eli Lilly, Janssen

DOI: 10.1136/annrheumdis-2019-eular.4091
AB0195

THE IMPACT OF KETOGENIC DIET AND HIGH-FATHIGH-GLUCOSE DIET IN PRISTANE INDUCED LUPUSLIKE NEPHRITIS MURINE MODEL

Chao-Yi Wu' ${ }^{1}$, Huang-Yu Yang ${ }^{2}$, Jing-Long Haung ${ }^{1} .{ }^{1}$ Linkou Chang Gung Memorial Hospital Hospital, Department of Pediatrics. Division of Allergy, Asthma, and Rheumatology, Taoyuan city, Taiwan, Republic of China; ${ }^{2}$ Linkou Chang Gung Memorial Hospital Hospital, Department of Nephrology, Taoyuan city, Taiwan, Republic of China

Background: Systemic lupus erythematous is a chronic systemic inflammatory disease which commonly involes kidneys[1]. Aside from the use of conventional immunosuppressants, dietary components and metabolites may likely affect our immune system.

Objectives: An interest in the anti-inflammatory property of ketogenic diet (KD) has recently come to attention.

It not only alters the balance between Th17 and Treg cells[2], its metabolite, beta-hydroxybutyrate was known to block NLRP3 inflammasomemediated inflammation[3-6]. High-fat-high-glucose diet (HFGD), on the other hand, was known for a proinflammatory property. Aim to understand the immune modulatory effect of KD and HFGD in cases with systemic lupus erythematous, a chronic systemic inflammatory disease with glomerulonephritis, pristane induce lupus like nephritis murine model was used. Methods: Pristane induced lupus nephritis mice were divided in to groups fed with regular chow (CD), KD and HFGD along with healthy controls The diets were kept for 6 months with regular body weight and urine protein monitoring. Serum samples were collected for metabolic evaluation and immune survey bimonthly. The mice were sacrificed 6 months after diet change. Kidneys, lymph nodes, spleen, blood and guts were collected for evaluation.

Results: KD and HFGD were both well tolerated by experimental mice Two months after diet change, higher level of beta-hydroxybutyrate and triglyceride but lower sugar level was noted in mice fed on KD when compared to those fed on CD and HFGD (all $p<0.05$ ). Mice fed on KD and HFGD have a much lower RBC and platelet count than those fed on $C D$ in the experimental mice group (both $p<0.05$ ). Although global lymphocyte counts were much lower in those pristine treated mice, Th17 lymphocytes were significantly higher in the blood as well as kidneys among those fed on HFGD (all $p<0.05$ ). This is compatible with their high serum concentration of anti-dsDNA, anti-nRNP and anti-Sm and the rapid progressing proteinuria. Renal, hepatic and intestinal histopathology was still under analysis at present.

Conclusion: In conclusion, food plays a critical role in immune modification. Despite the reported anti-inflammatory effect of KD, it does not mitigate lupus nephritis progression. HFGD formula, however, accelerated the autoimmune phenotype for cases with lupus like glomerulonephritis.

\section{REFERENCES:}

[1] Borchers AT, Leibushor N, Naguwa SM, Cheema GS, Shoenfeld Y, Gershwin ME (2012) Lupus nephritis: a critical review. Autoimmun Rev 12:174-194.

[2] Ni FF, Li CR, Liao JX, Wang GB, Lin SF, Xia Y, Wen JL (2016) The effects of ketogenic diet on the Th17/Treg cells imbalance in patients with intractable childhood epilepsy. Seizure 38:17-22.

[3] Goldberg EL, Asher JL, Molony RD, Shaw AC, Zeiss CJ, Wang C, Morozova-Roche LA, Herzog RI, Iwasaki A, Dixit VD (2017) beta-Hydroxybutyrate Deactivates Neutrophil NLRP3 Inflammasome to Relieve Gout Flares. Cell Rep 18:2077-2087.

[4] Choi IY, Piccio L, Childress P, Bollman B, Ghosh A, Brandhorst S, Suarez J, Michalsen A, Cross AH, Morgan TE, Wei M, Paul F, Bock M, Longo VD (2016) A Diet Mimicking Fasting Promotes Regeneration and Reduces Autoimmunity and Multiple Sclerosis Symptoms. Cell Rep 15:2136-2146.

[5] Storoni M, Plant GT (2015) The Therapeutic Potential of the Ketogenic Diet in Treating Progressive Multiple Sclerosis. Mult Scler Int 2015:681289.

[6] Choi IY, Lee C, Longo VD (2017) Nutrition and fasting mimicking diets in the prevention and treatment of autoimmune diseases and immunosenescence. Mol Cell Endocrinol 455:4-12.

Disclosure of Interests: None declared

DOI: 10.1136/annrheumdis-2019-eular.1102 